\title{
A 4D IMRT planning method using deformable image registration to improve normal tissue sparing with contemporary delivery techniques
}

\author{
Xiaogiang Li, Xiaochun Wang, Yupeng Li and Xiaodong Zhang
}

\begin{abstract}
We propose a planning method to design true 4-dimensional (4D) intensity-modulated radiotherapy (IMRT) plans, called the t4Dplan method, in which the planning target volume (PTV) of the individual phases of the 4D computed tomography $(\mathrm{CT})$ and the conventional PTV receive non-uniform doses but the cumulative dose to the PTV of each phase, computed using deformable image registration (DIR), are uniform. The non-uniform dose prescription for the conventional PTV was obtained by solving linear equations that required motion-convolved 4D dose to be uniform to the PTV for the end-exhalation phase (PTV50) and by constraining maximum inhomogeneity to $20 \%$. A plug-in code to the treatment planning system was developed to perform the IMRT optimization based on this non-uniform PTV dose prescription. The 4D dose was obtained by summing the mapped doses from individual phases of the 4D CT using DIR. This 4D dose distribution was compared with that of the internal target volume (ITV) method. The robustness of the 4D plans over the course of radiotherapy was evaluated by computing the $4 \mathrm{D}$ dose distributions on repeat 4D CT datasets. Three patients with lung tumors were selected to demonstrate the advantages of the t4Dplan method compared with the commonly used ITV method. The 4D dose distribution using the t4Dplan method resulted in greater normal tissue sparing (such as lung, stomach, liver and heart) than did plans designed using the ITV method. The dose volume histograms of cumulative 4D doses to the PTV50, clinical target volume, lung, spinal cord, liver, and heart on the 4D repeat CTs for the two patients were similar to those for the 4D dose at the time of original planning.
\end{abstract}

Keywords: 4D CT, IMRT, treatment planning, respiratory motion, deform

\section{Introduction}

Implementations of four-dimensional (4D) radiotherapy based on 4D computed tomography (CT) datasets have been described by Rietzel et al [1] and Keall [2]. In 4D radiotherapy, the treatment plan is designed on each $4 \mathrm{D}$ CT image set (i.e., 4D treatment planning), and radiation is delivered throughout the patient's breathing cycle (i.e., 4D treatment delivery), which ensures adequate coverage of the tumor target without increasing the treated volume. Because 4D treatment planning accounts for temporal changes in anatomy, 4D radiotherapy holds promise as the optimal method for treating patients. However, 4D radiotherapy currently requires $4 \mathrm{D}$ treatment delivery, which necessitates

\footnotetext{
* Correspondence: xizhang@mdanderson.org

Department of Radiation Physics, The University of Texas, MD Anderson Cancer Center, Houston, Texas 77030, USA
}

sophisticated device(s) to synchronize the treatment delivery with the patient's respiration. Most centers have the ability to acquire 4D CT images, but they do not have the ability to perform $4 \mathrm{D}$ radiation delivery. Instead, 4D CT images are primarily used to define the internal target volume (ITV), which is essentially the envelope needed to enclose the target as it moves throughout the breathing cycle. 4D CT [3-9] provides a more accurate tumor volume definition since it limits motion artifacts during CT acquisition, displays the anatomically correct shape and size of the tumor, and demonstrates respiration-induced motion of the tumor and organs at risk. Previous studies using 4D CT datasets have mostly been focused on dosimetric verification to determine if dose distribution planned on one or part of the 4D CT datasets is adequate to estimate the cumulative dose from all 4D CT datasets $[1,10]$. Few studies 
have investigated whether the information on anatomic motion provided by 4D CT can be used to design treatment plans that confer the advantages of $4 \mathrm{D}$ treatment delivery without requiring additional equipment.

In this paper, we describe an effective and practical 4D treatment planning method, which we refer to true 4D planning (t4Dplan) method, for intensity-modulated radiotherapy (IMRT) using 4D CT datasets to maximize critical structure sparing. In traditional treatment planning, the prescribed dose is planned to be distributed uniformly to the target while minimal dose is delivered to the surrounding normal structures on the planning $\mathrm{CT}$ under the assumption that the planning $\mathrm{CT}$ truly represents the patient anatomy that will be present during treatment. In our t4Dplan method, however, planning deliberately creates non-uniform dose distribution in the target (i.e., it creates hot regions along the target's direction of motion on the planning CT) to achieve a uniform dose distribution in the target and minimal dose to the surrounding normal structures on the final 4D dose distribution. The difference between the t4Dplan method and the traditional ITV method is illustrated in figure 1. The t4Dplan method does not require $4 \mathrm{D}$ treatment delivery and is solely dependent on the $4 \mathrm{D}$ datasets acquired during the planning process. Compared to some other techniques such as respiratory gating [11], breath hold [12,13] and dynamic MLC tumor tracking [14-16], the t4Dplan method is easier to implement in the clinic because it uses the current treatment planning and delivery systems.

\section{Materials and methods \\ 2.1. t4Dplan}

The t4Dplan method, which uses 4D CT datasets, designs treatment plans as follows:

1. A reference CT dataset is selected from all the 4D CT datasets. Usually, an end-of-exhalation phase CT (i.e., the $50 \%$ phase [T50]) is selected as the reference CT dataset [17] since patients spend more time at the end of exhalation [18].

2. The target volume (TV) is outlined based on the reference $\mathrm{CT}$.

3. The motion TV (MTV) is outlined on the reference $\mathrm{CT}$ as the combined volume of the target at all phases of the 4D CT datasets (i.e., the MTV is an envelope enclosing the target as it moves throughout the breathing cycle).

The t4Dplan method calculates a deliverable non-uniform dose distribution (i.e., the apparent dose distribution [AppD]) to the MTV. The final 4D dose distribution is determined by recalculating the t4Dplan on each phase of the 4D CT dataset and creating a time-averaged cumulative dose distribution based on deformable image registration (DIR).

For each voxel on the reference CT, the corresponding voxel on another phase of the CT dataset can be derived through DIR by transforming the source image (i.e., the reference CT) to the target image (i.e., another phase of the CT dataset), such that

$$
v_{i}^{j}=T^{j, r e f} \times v_{i}^{r e f},
$$

where $v_{i}^{\text {ref }}$ is the position vector for the $i$ th voxel on the reference $\mathrm{CT}, T^{j}$,ref represents the transform matrix from the reference CT to the $j$ th phase of the CT dataset, and $v_{i}^{j}$ is the position vector for the corresponding voxel on the $j$ th phase of the CT dataset for the $i$ th voxel on the reference CT.

In the current study, to derive the non-uniform dose, we first assumed that the dose on each phase of the $4 \mathrm{D}$ CT was approximately the same as the AppD on the reference CT. This approximation assumes the internal movement of anatomy will not impact the dose distribution and is a good approximation for photon dose calculation. It should be noted that this approximation is only used in the derivation of a non-uniform dose prescription. For the final designed plan, we used the exact 4D dose calculation without this approximation. The 4D dose for each voxel on the reference CT can be approximated as the time-averaged cumulative dose of the corresponding voxel on all phases in the CT dataset, such that

$$
D^{4 D}\left(v_{i}^{r e f}\right)=\frac{1}{K} \sum_{j=1}^{K} D^{A p p D}\left(v_{i}^{j}\right),
$$

where $K$ represents the number of phases of the CT datasets, $D^{4 D}\left(v_{i}^{\text {ref }}\right)$ is the $4 \mathrm{D}$ dose for the $i$ th voxel on the reference CT, and $D^{A p p D}\left(v_{i}^{j}\right)$ is the AppD for the corresponding voxel on the $j$ th phase of the dataset.

Assuming the MTV and TV on the reference CT have $n$ and $m(n>m)$ voxels, respectively, and the AppD values for the $n$ voxels of the MTV are $D_{1}, D_{2}, \ldots, D_{n}$, the 4D dose distribution for the TV with $m$ voxels can be determined using the following linear equations derived from equation (2):

$$
\begin{aligned}
& D^{4 D}\left(v_{1}^{r e f}\right)=\frac{1}{K}\left(D^{A \rho p D}\left(v_{1}^{1}\right)+D^{A p D}\left(v_{1}^{2}\right)+\ldots \ldots .+D^{A p D D}\left(v_{1}^{K}\right)\right)=D_{0}, \quad 1^{\text {st }} \text { voxel; } \\
& D^{4 D}\left(v_{2}^{\text {ref }}\right)=\frac{1}{K}\left(D^{A \rho p D}\left(v_{2}^{1}\right)+D^{A \rho p D}\left(v_{2}^{2}\right)+\ldots . . .+D^{A \rho p D}\left(v_{2}^{K}\right)\right)=D_{0}, \quad 2^{\text {nd }} \text { voxel; } \\
& D^{4 D}\left(v_{m}^{\text {ref }}\right)=\frac{1}{K}\left(D^{A \rho p D}\left(v_{m}^{1}\right)+D^{A \rho p D}\left(v_{m}^{2}\right)+\ldots . .+D^{A \rho p D}\left(v_{m}^{K}\right)\right)=D_{0}, \quad m \text { th voxel, }
\end{aligned}
$$

where $D^{A p p D}\left(v_{i}^{j}\right)=D_{1}, D_{2}, \ldots, \quad$ or $D_{n}$, are the unknown parameters, and $D_{0}$ is the uniform dose prescribed to the TV (i.e., the final 4D dose distribution on the TV). Here, we have $n$ unknown parameters (i.e., $D_{1}$, 




T4Dplan method



ITV method

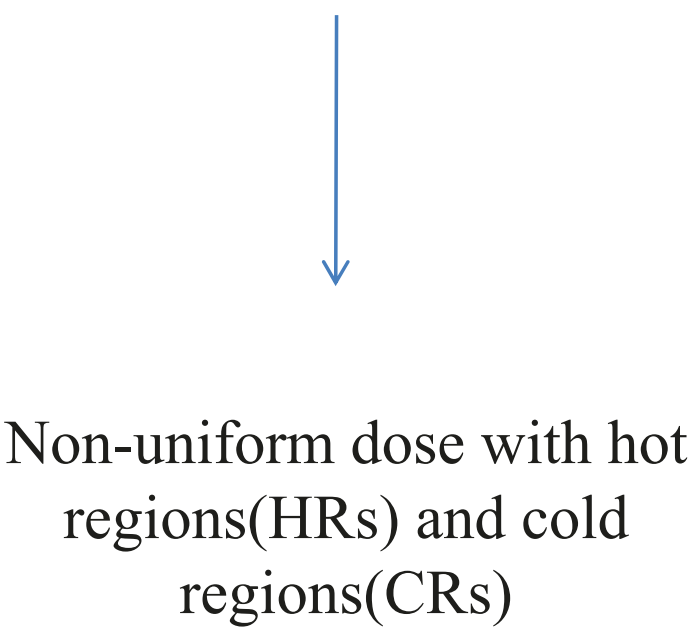

(a) (b)

\section{Uniform dose with larger area}

Figure 1 The difference between (a) t4Dplan method and (b) traditional ITV method. The planning target volume (PTV) in the ITV method is the target volume used to plan and treat. In the t4Dplan method, the PTV50 plus the hot regions (HRs) are the target volume used to plan and treat. The cold regions (CRs) in the t4Dplan method represent the reduced treated volume relative to that in plans from the ITV method. CTV represents the clinical target volume; GTV represents gross tumor volume; IGTV represents internal gross tumor volume.

$\left.D_{2}, \ldots, D_{n}\right)$ that need to be derived from $m$ equations, with $m<n$. The solution for equation group (3) is underdetermined. In order to clarify the idea how the linear equations are constructed and the non-uniform dose distribution is derived more clearly, we used a simple phantom (shown in figure 2) to illustrate. This phantom shown in figure $2(\mathrm{a}, \mathrm{b}, \mathrm{c}, \mathrm{d}) \mathrm{had} 4$ phases, the MTV had 4 voxels (voxel $1-4$ ), the TV had 2 voxels (shadow area). So the linear equations were constructed by $4 \mathrm{D}$ dose convolution of each TV voxel as follows:

$$
\begin{array}{ll}
D^{4 D}\left(v_{1}^{r e f}\right)=\left(\frac{1}{4} D_{1}+\frac{1}{2} D_{2}+\frac{1}{4} D_{3}\right)=D_{0}, & 1^{\text {st }} \text { TV voxel } \\
D^{4 D}\left(v_{2}^{r e f}\right)=\left(\frac{1}{4} D_{2}+\frac{1}{2} D_{3}+\frac{1}{4} D_{4}\right)=D_{0}, & 2^{\text {nd }} \text { TV voxel }
\end{array}
$$




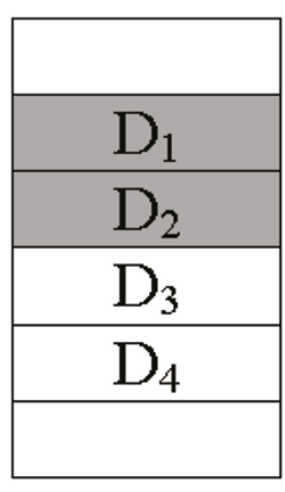

(a)

\begin{tabular}{|c|}
\hline \\
\hline 0 \\
\hline $4 \mathrm{D}_{0} / 3$ \\
\hline $4 \mathrm{D}_{0} / 3$ \\
\hline 0 \\
\hline
\end{tabular}

(e)

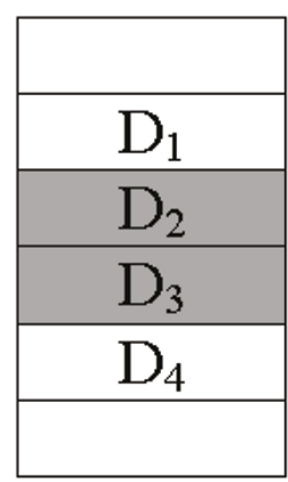

(b)

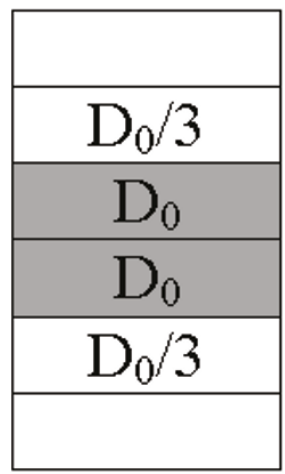

(f)



(c)



(g)

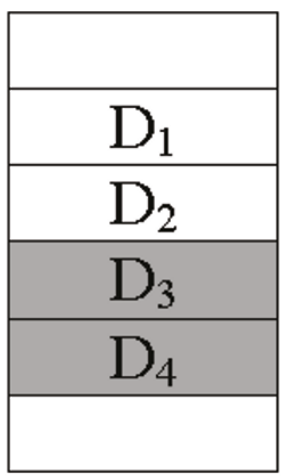

(d)

\begin{tabular}{|c|}
\hline $\mathrm{D}_{0} / 4$ \\
\hline $3 \mathrm{D}_{0} / 4$ \\
\hline $\mathrm{D}_{0}$ \\
\hline $\mathrm{D}_{0}$ \\
\hline $3 \mathrm{D}_{0} / 4$ \\
\hline $\mathrm{D}_{0} / 4$ \\
\hline
\end{tabular}

(h)

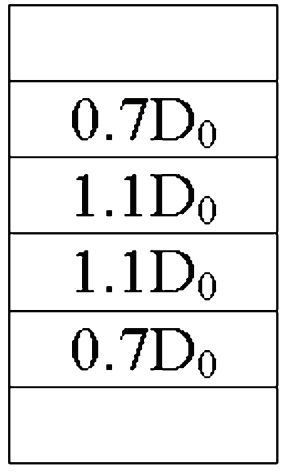

(i)

\begin{tabular}{|c|}
\hline $0.175 \mathrm{D}_{0}$ \\
\hline $0.625 \mathrm{D}_{0}$ \\
\hline $\mathrm{D}_{0}$ \\
\hline $\mathrm{D}_{0}$ \\
\hline $0.625 \mathrm{D}_{0}$ \\
\hline $0.175 \mathrm{D}_{0}$ \\
\hline
\end{tabular}

(j)

Figure $2 \mathrm{~A}$ phantom with tumor volume (shadow area) moving only in superior-inferior direction was illustrated to show how the non-uniform dose distribution was derived. The moving circle was divided into 4 phases (a), (b), (c), (d). The non-uniform prescribed dose distribution derived by the t4Dplan method was shown on (e) and its corresponding 4D dose was shown on (f) by summing the dose for 4 phases evaluated on (b). The prescribed dose for ITV approach (g) and the 4D dose (f). The non-uniform dose distribution acquired for t4Dplan with the total variation regulation from formula (5) was shown in (i) and its corresponding 4D dose (j). 
Since the linear equations had 4 unknown parameters and only 2 equations, it was undetermined. One of the solutions can be acquired by applying an extra constraint, which implied the smallest margin $\left(D_{1}=D_{4}=\right.$ 0 ), and was shown figure 2(e). The corresponding $4 \mathrm{D}$ dose referenced on the second phase (figure 2(b)) was calculated and shown on figure 2(f). Compared to the ITV approach, distributing uniform prescribed dose to all the voxels (figure $2(\mathrm{~g})$ ), resulting $4 \mathrm{D}$ dose (figure 2 (h)) when accumulating for all phases, the non-uniform dose distribution clearly decreased the margin, spared the critical organ while maintaining the same target coverage (figure $2(\mathrm{f})$ vs figure $2(\mathrm{~h})$ ).

The non-uniform dose as shown is figure 2(e) was an ideal prescribed dose, assuming the dose can be delivered for this pattern. In reality, when delivering a high dose to a specific voxel, it is impractical to achieve a very low dose in the nearby voxels due to the dose falloff gradient. For this reason, one possible AppD can be acquired by minimizing the following objective function with a total-variation regulation [19]:

$$
\begin{aligned}
& X=\sum_{i}^{n} D^{A p p D}\left(v_{i}^{r e f}\right)+\lambda \sum_{i}^{n}\left(D^{A p p D}\left(v_{i}^{r e f}\right)-D^{A p p D}\left(v_{i-1}^{r e f}\right)\right)^{2}, \\
& \text { subject to (2): } D^{4 D}\left(v_{i}^{r e f}\right)=\frac{1}{K} \sum_{j=1}^{K} D^{A p p D}\left(v_{i}^{j}\right) \geq D_{0}, \\
& \qquad 120 \% \times D_{0} \geq D_{i} \geq 0, D_{i}=D_{1}, D_{2}, \ldots, D_{n},
\end{aligned}
$$

where $n$ is the total number voxels of MTV on the reference CT dataset. The parameter $\lambda$ is the importance factor to penalize the second term of (5) which calculates the sum of absolute derivatives. The penalty tends to have zero derivatives and smoothes the voxels' prescribed doses for easy delivery. The formula (6) constrains the maximum inhomogeneity to be $20 \%$. The reason is that when we create the apparent plan with designed hot region, we want the apparent plan not to be too hot. In our routine clinical practice, our clinician sometimes also accepts the plan $20 \%$ hot in the PTV, therefore, the plan can be readily used in the routine clinical practice. For the phantom in figure 2, the solution to minimize equation (5) was shown in figure 2(i). Compared to the ideal solution (figure 2(e)), the solution with the total-variation regulation blurred the non-uniform dose and created a more natural dose fall-off, which became practical for deliverable optimization. The corresponding non-uniform $4 \mathrm{D}$ dose, as shown in figure 2(j), still had enough dose coverage to the target and more normal tissue sparing than that of the ITV approach (figure 2(j) vs figure $2(\mathrm{~h})$ ).

The derived AppD for the MTV served as the nonuniform dose prescription for the MTV. The deliverable AppD can be obtained using an IMRT optimization system. The voxel-based optimization function was used to achieve the AppD for the MTV on reference CT, such that

$$
f=\sum_{i=1}^{n}\left(D\left(v_{i}^{r e f}\right)-D^{A p p D}\left(v_{i}^{r e f}\right)\right)^{2},
$$

In other words, the derived AppD for the MTV becomes the optimization objective for the MTV. The Pinnacle treatment planning system (version $8.1 \mathrm{x}$, Philips Medical Systems, Milpitas, CA) was used as the platform for IMRT optimization. The in-house-developed plug-in module, which optimizes the dose distribution to achieve the derived AppD for the MTV using equation (7), cooperates with the Pinnacle IMRT optimization system to achieve the final deliverable AppD on the reference $\mathrm{CT}$, which results in a uniform dose to the TV and minimal dose to the surrounding critical structures for $4 \mathrm{D}$ dose distribution. In our implementation, only the objective function (7) was added to the Pinnacle inverse planning module as a plug-in. The conventional objectives that are not voxelized can still be used to control normal tissue sparing and target coverage. From the treatment planner's point of view, our method is an enhancement of the current planning method. This implementation makes our method readily available for use in routine clinical practice.

Once treatment optimization based on the AppD was obtained, the dose on each 4D CT was recalculated and the $4 \mathrm{D}$ dose distribution obtained by using DIR.

\subsection{Evaluation of t4Dplan method}

Three patients with tumor located in the middle lobe of the right lung (patient 1), near the diaphragm of the left lung (patient 2) and near the diaphragm of the right lung (patient 3) respectively were selected for our evaluation of the t4Dplan method. The characteristics of the three patients were listed in Table 1. All patients had been enrolled on an institutional review board approved protocol and treated at The University of Texas MD Anderson Cancer Center. According to the protocol, the 4D CT datasets for each patient had been acquired in 2.5- $\mathrm{mm}$ slices using a multislice CT scanner (Discovery ST, General Electric Healthcare Systems, Waukesha, WI) and the Real-Time Position Management (RPM) respiratory gating system (Varian Medical Systems, Palo Alto, CA). Ten CT datasets corresponding to the 10 phases in each equally divided respiration cycle (from the $0 \%$ phase, referred as the T0 CT, to the $90 \%$ phase, referred as the T90 CT) were reconstructed [20] for each 4D dataset. The end-of-exhalation phase CT (i.e., T50 CT) dataset from the 4D dataset acquired during simulation was selected as the reference and planning CT set. The TV was defined as the planning 
Table 1 Characteristics of the three patients used in the study.

\begin{tabular}{cccccc}
\hline Patient & GTV50 $\left(\mathbf{c m}^{\mathbf{3}}\right)$ & PTV $\left(\mathbf{c m}^{3}\right)$ & Center-to-center tumor motion $(\mathbf{c m})$ & Primary tumor motion direction & Prescribed dose $(\mathbf{f x s} \times$ Gy/fx) \\
\hline 1 & 61.14 & 481.15 & 2.65 & S-l & $35 \times 1.8$ \\
2 & 156.9 & 878.7 & 1.53 & S- & $35 \times 1.8$ \\
3 & 218.108 & 1204.06 & 3.42 & S-l & $35 \times 1.8$ \\
\hline
\end{tabular}

Abbreviations: $\mathrm{fx}(\mathrm{s})=$ fraction $(\mathrm{s})$

treatment volume (PTV) on the reference CT (i.e., PTV50), which was generated by defining the gross tumor volume (GTV) on the reference CT and then expanding the GTV by $8 \mathrm{~mm}$ to obtain the clinical TV (CTV) on the reference CT (i.e., CTV50) and by another $8-\mathrm{mm}$ to obtain the PTV50. The MTV was defined as the PTV that was generated by a $16-\mathrm{mm}$ expansion of the combined volume of the GTVs at all 10 phases, named the internal GTV (IGTV), i.e., 8-mm expansion of the IGTV to obtain the ITV and another $8-\mathrm{mm}$ expansion of the ITV to obtain the PTV. The margins to expand from IGTV to ITV and ITV to PTV are currently adopted as the standard for the thoracic patients in our institution [21]. The prescription specified that the $4 \mathrm{D}$ dose (i.e., $\mathrm{D}_{0}$ ) of 63 Gy covers at least $95 \%$ of the PTV50 on the reference CT.

The t4Dplan method was used to design the treatment plans for all patients. The non-uniform AppD for the PTV was derived from equations (3), (5), and (6), and the deliverable AppD was achieved by optimization using equation (7). The clinical treatment plan for all patients had been designed by our experienced dosimetrists based on the commonly used ITV method (i.e., the IMRT plan was designed to have uniform dose distribution to the PTV and minimal dose to normal tissues.). In this study, we re-optimized those plans and found that those plans could not be improved upon to our best effort and knowledge. To compare the plan based on the t4Dplan method with the plan based on the ITV method, the dose volume histograms (DVHs) for the PTV50 and normal structures (i.e., total lung, stomach, liver, spinal cord, and heart) were calculated based on deliverable AppD and 4D dose distribution. To assess plan quality with respect to target dose, we computed the conformity number $(\mathrm{CN})$ for the PTV50 using the following definition [22]:

$$
C N=\frac{T V_{D p}}{T V} \times \frac{T V_{D p}}{V_{D p}},
$$

where $T V_{D p}$ is the TV covered by the prescribed dose and $V_{D p}$ is the total volume enclosed by the prescription isodose surface. The $\mathrm{CN}$ ranges from 0 to 1 , and the greater the $\mathrm{CN}$, the better the prescribed dose's conformation to the target. A small $\mathrm{CN}$ indicates that either the target is not well covered by the prescribed dose (the first fraction of the equation) or the total volume of tissue receiving the prescribed dose was very large compared to the target (the second fraction of the equation).

The ratio $\left(R_{\text {prescribed_dose }}\right)$ of the prescribed dose volume in 4D dose distribution $\left(V_{4 D}\right)$ and deliverable AppD $\left(V_{A p p D}\right)$ was calculated for plans derived from both the t4Dplan method and the ITV method for each patient to show the dosimetric effects of respirationinduced organ motion:

$$
R_{\text {prescribed_dose }}=\frac{V_{4 D}}{V_{A p p D}} \text {. }
$$

\subsection{Robustness of the t4Dplan method against interfractional variation of the respiratory pattern}

To evaluate the robustness of t4Dplan method against the irregular breathing motion pattern, patient 2 and 3 were used. Since for these patients, not only $4 \mathrm{D}-\mathrm{CT}$ were obtained during simulation to allow consideration of tumor motion in planning, but also several repeat4D CTs were obtained to assess the intra- and inter-fractional movement of the target volumes and the normal structures. One repeat 4D CT datasets acquired during week 2 of treatment for patient 2 and week 3 for patient 3 were selected to evaluate the robustness of the t4Dplan against inter-fractional variation in the respiratory pattern. Figure 3 shows the right-left (RL), anteriorposterior (AP), and SI shifts of the GTV on each phase (i.e., T0, T10, ...) relative to the T50 phase for both the simulation CT and repeat CT for patient 2. The repeat 4D CTs were also registered to the simulation 4D dataset using bony structures, and figure 4 shows the anatomic changes between coronal CT images obtained at simulation and week 2 for patient 2. Both figures 3 and 4 demonstrate the irregularity of breathing motion during fractional radiation treatments.

To evaluate whether the non-uniform dose prescription derived solely on the 4D simulation CT could still provide good target coverage and normal tissue sparing if the breathing pattern was irregular during fractional treatments, we recalculated the AppD in each phase of the repeat $4 \mathrm{D}$ datasets based on the plans designed using simulation CT and bony registration. The 4D dose distribution was cumulated and displayed on the reference CT (T50) of the repeat CT. The DVHs for the PTV50 and normal structures were calculated based on 


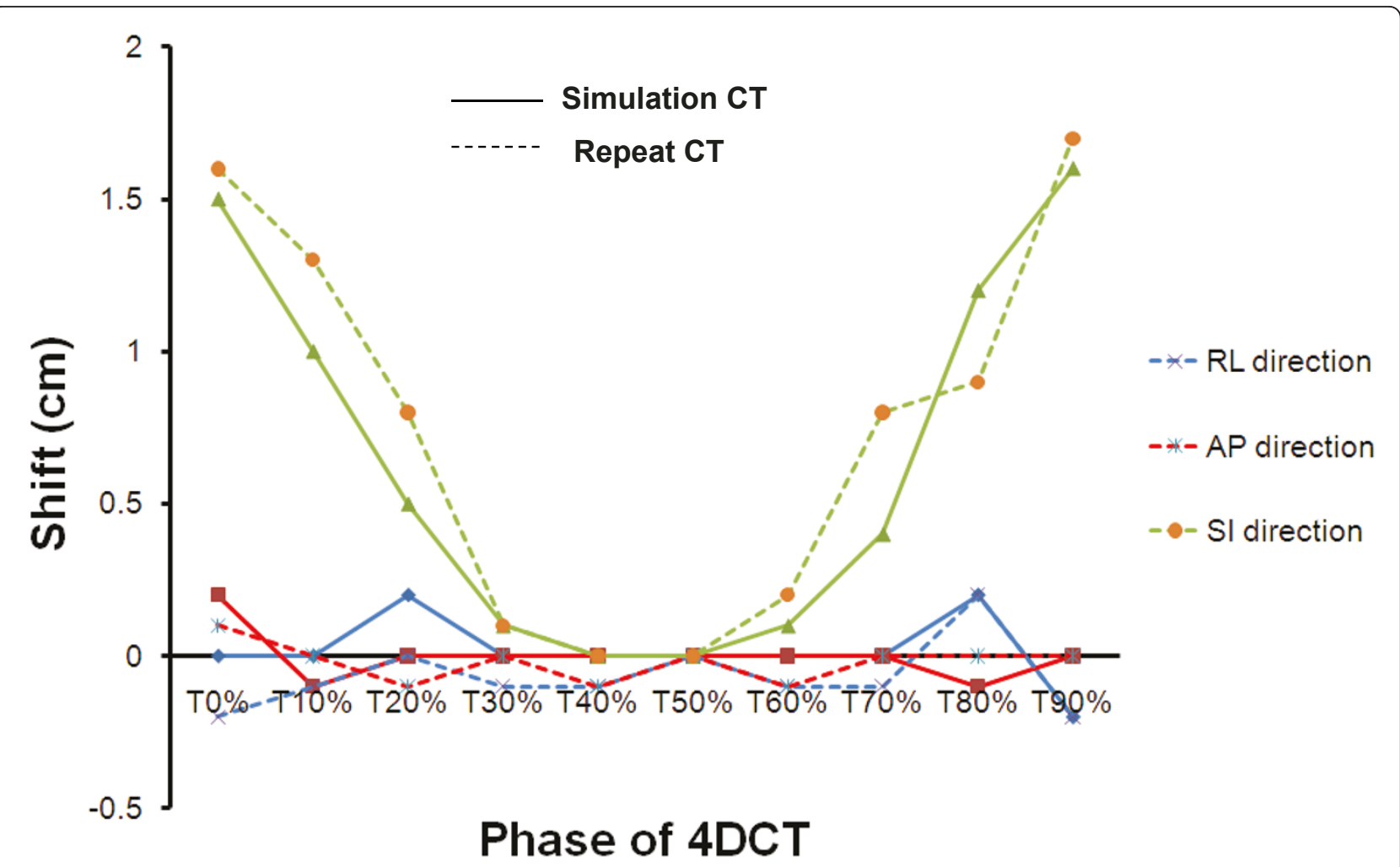

Figure 3 The GTV motion on the simulation CT datasets (solid line) and repeat CT datasets (dashed line) for 10 phases of the respiratory cycle relative to the T50 phase in the right-left (RL) direction (blue color), anterior-posterior (AP) direction (red color), and superior-inferior (SI) direction (green color) for patient 2.

the 4D dose distributions to show the effects of interfractional variation in the respiratory pattern of the patient in the t4Dplan method.

\section{Results}

\subsection{Theoretic and deliverable AppD}

Figure 5 shows the theoretic and deliverable AppD for patient 1 (figures $5(\mathrm{a})$ and $5(\mathrm{~b})$ ), patient 2 (figures $5(\mathrm{c}$ ) and $5(\mathrm{~d})$ ) and patient 3 (figures $5(\mathrm{e})$ and $5(\mathrm{f})$ ). The theoretic AppD hot regions (i.e., $70 \mathrm{~Gy}$, red color-wash on figure 5(a), (c) and 5(e)) for the PTV were located inferior to the PTV50 for all patients. Tumor motion in SI direction was $2.6 \mathrm{~cm}$ for patient $1,1.5 \mathrm{~cm}$ for patient 2 and $3.31 \mathrm{~cm}$ for patient 3; motion in AP direction was $0.2 \mathrm{~cm}$ for patient 1 and $2,0.85 \mathrm{~cm}$ for patient 3 ; and motion in RL direction was $0.5 \mathrm{~cm}$ for patient $1,0.2 \mathrm{~cm}$ for patient 2. The SI direction was the dominant direction of tumor motion for all patients, which resulted in the hot regions of theoretic AppD appearing along the SI direction.

The optimized deliverable AppD was similar to the theoretic AppD for all patients, with the hot regions (i.e. 70 Gy isodose line in figure 5(b), figure 5(d) and figure 5(f)) mainly located inferior to the PTV50. The more similar the deliverable AppD was to the theoretic AppD, the more uniform the $4 \mathrm{D}$ dose distribution was in the PTV50 on the reference CT.

\subsection{Normal-structure sparing in the t4Dplan method}

Table 2 lists all the dosimetric indices for the 4D dose distributions calculated using the t4Dplan method and ITV method for all three patients. Since the tumor located in the middle lobe of the right lung for patient 1 , the total lung sparing was significant using t4Dplan method compared to ITV method. For other two patients, as tumor located in the lower lobe of lung near the diaphragm and close to stomach (patient 2) or liver (patient 3), significant dose reduction for stomach and liver were observed using t4Dplan method compared to ITV method, respectively. And the total lung sparing was comparable using two methods. The reduction of the mean dose of heart of 4D dose distributions for all the three patients using t4Dplan method, were $3 \mathrm{~Gy}, 0.4$ Gy and 0.2 Gy, respectively, from that using ITV method. The maximum cord dose of $4 \mathrm{D}$ dose distributions for patient 1 was slightly higher using t4Dplan method than that using ITV method, but far lower than cord tolerate dose (i.e. 45 Gy). 


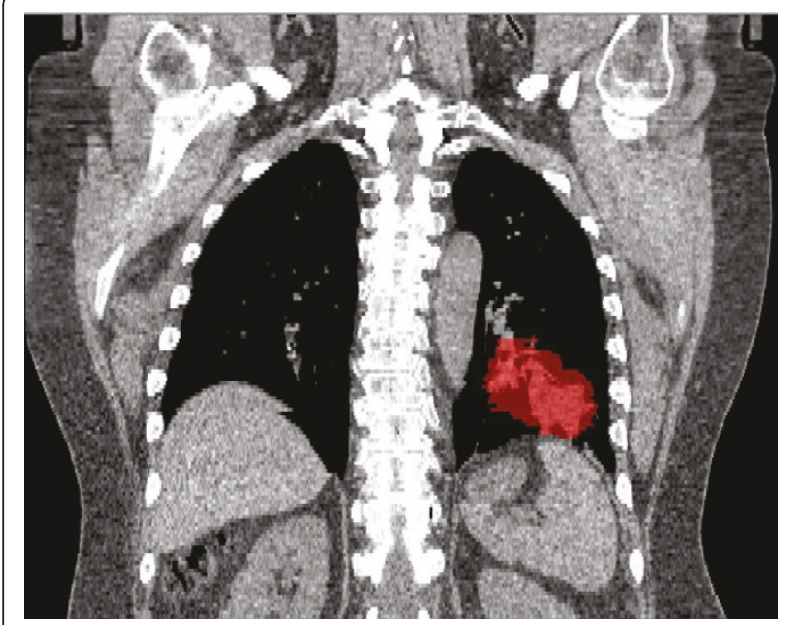

\section{Simulation CT}

(a)

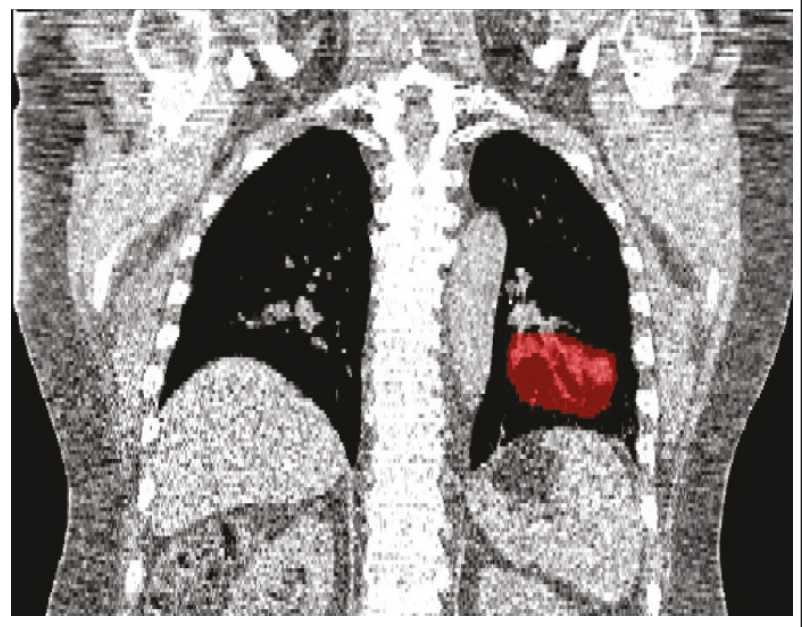

Repeat CT

\section{(b)}

Figure 4 Changes of GTVs (red color-wash) and other anatomic structures on coronal view of (a) simulation CT datasets and (b) repeat CT datasets for patient 2

The PTV50 coverage by the 63 Gy prescribed dose (V63) for 4D dose distribution using the t4Dplan method was inferior to the coverage obtained using the ITV method from table 2. However, the $\mathrm{CN}$ for all patients using the t4Dplan method was significantly better than the $\mathrm{CN}$ of the ITV method. It indicates that the ITV method overestimated the TV. In other words, the ITV method overestimates the dose effect of respiration-induced target motion. Consequently, a large volume of normal tissue will be unnecessarily irradiated if the ITV method is used.
Figure 6 shows the 4D dose distributions calculated using the t4Dplan method and ITV method for the three patients respectively. The high-dose isodose lines (such as 63 Gy and 60 Gy) spread out especially in inferior direction for ITV method and conformed to the target very well for t4Dplan method. The low-dose isodose line pushed out slightly from target for ITV method compared to $4 \mathrm{D}$ paln method. It illustrates more normal tissue sparing using t4Dplan method than ITV method.

The $R_{63}$ which is the ratio of volume greater than or equal to 63 Gy (prescription) of 4D cumulative dose distribution to apparent dose distribution is listed in table 2 . They were all less than 1 , meaning that target motion effectively smears the dose and reduces the high-dose volume. Comparing deliverable AppD and 4D dose distributions (i.e., figure 5(b) vs figure 6(a) for patient 1 , figure $5(\mathrm{~d})$ vs figure $6(\mathrm{c})$ for patient 2 and figure $5(\mathrm{f})$ vs figure $6(\mathrm{e})$ ), it shows the prescribed isodose line (i.e., 63 Gy) on the $4 \mathrm{D}$ dose distribution was pushed in the superior direction and that it conformed well to the PTV50 (figures 6(a), (c) and 6(e)), since the dominant motion of the target was in the SI direction; hot regions (i.e., $\geq 70$ Gy) were smeared out and significantly smaller on 4D dose distribution than that on the deliverable AppD. On the contrary, ITV method overestimated the target motion and the prescribed isodose line in ITV method enclosed many healthy lung tissues (figure 6(b), (d) and 6(f)). This fact is also reflected in CN index.

Since respiratory motion effectively reduces the volume receiving a high dose, as mentioned above, it may cause under dosing in the target if the plan is not designed to compensate for the motion-induced effects. Conversely, respiratory motion will create a more uniform target dose than designed if the plan is designed to compensate for the motion-induced effects.

\subsection{Robustness of the t4Dplan method}

Figure 7 shows the DVHs and 4D dose distributions calculated on the simulation $\mathrm{CT}$ and repeat $\mathrm{CT}$ datasets for patient 2 and 3 (there was no 4D repeat CT available for patient 1). The coverage of CTV50 and PTV50 on the repeat CT was as good as that on the simulation CT for patient 2 and a little better than that on the simulation CT for patient 3. The DVHs of the normal structures were similar between the two distributions from simulation $\mathrm{CT}$ and repeat $\mathrm{CT}$ for both patients. This result indicates that there were essentially no significant changes in dose distribution for the plan designed using the 4 Dplan even if there are some irregularities of respiration pattern for the patient from week to week. The stomach received fewer doses during week 2 of the treatment because volume of stomach was reduced during week 2 for patient 2 . In other words, the t4Dplan 


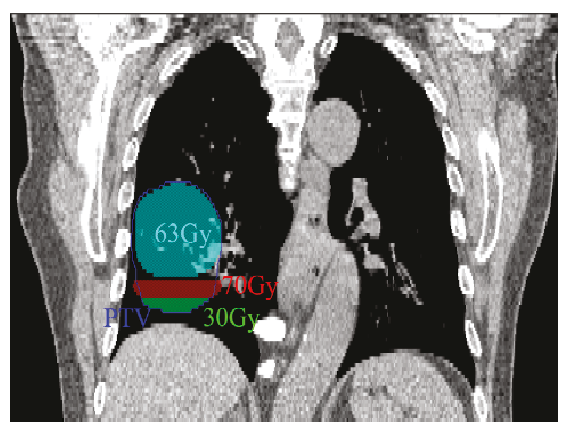

Theoretic AppD

(a)

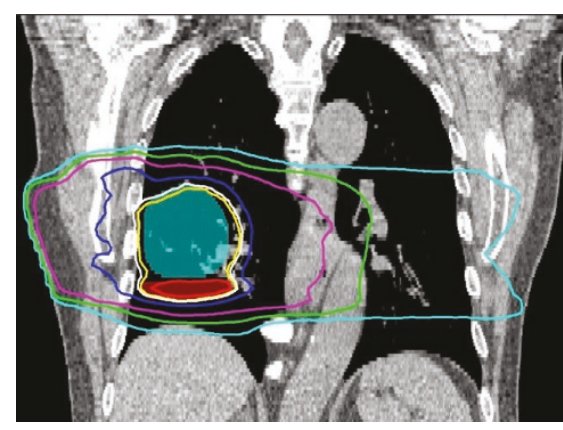

Deliverable AppD

(b)

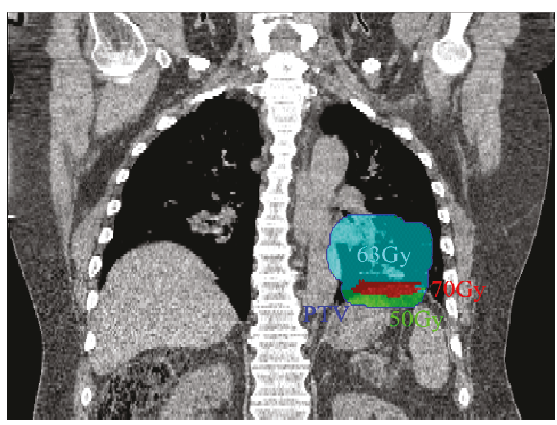

Theoretic AppD

(c)

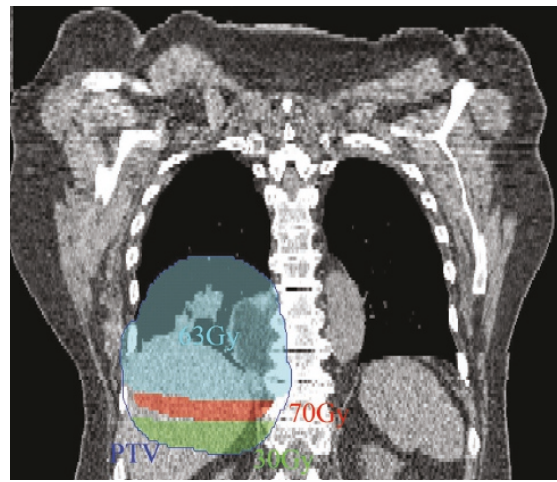

Theoretic AppD

(e)
Patient 1

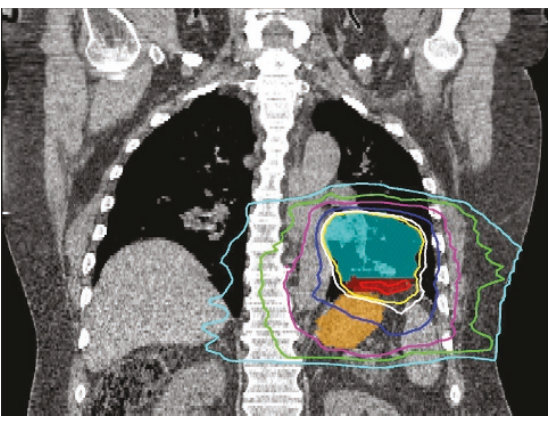

Deliverable AppD

(d)

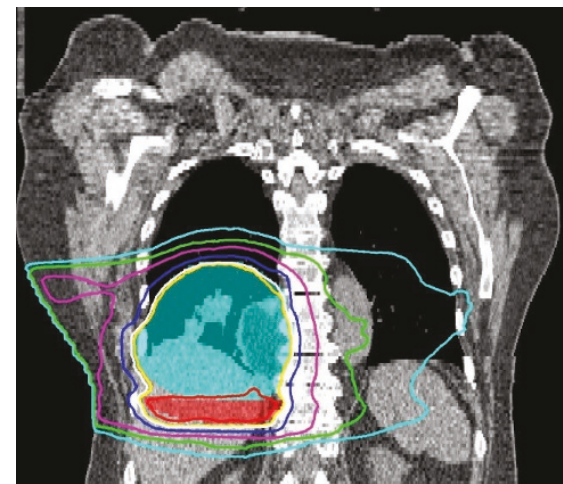

Deliverable AppD

(f)

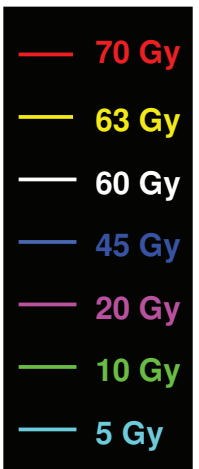

Figure 5 The theoretic and deliverable AppDs for patient 1 shown on panel (a) and panel (b), for patient 2, shown on panel (c) and panel (d) and for patient 3, shown on panel (e) and panel (f) respectively. The red and green color-wash on panel (a), (c) and (e) represent hot region (i.e. 70 Gy) and cold region (i.e. 30 Gy for patient 1 and 3, 50 Gy for patient 2) respectively for theoretic AppD. The blue color-wash on all the panels represents PTV50. The orange color-wash on (d) represents the stomach for patient 2.

method is robust against inter-fractional variation in the respiratory pattern.

\subsection{Planning time for t4Dplan}

Currently, the t4Dplan was implemented as a plug-in to Pinnacle (8.1x), which runs on an AMD Opteron 8220
CPU operating at $2800 \mathrm{MHz}$ with an i387-compatible floating-point operation processor. It took less than 10 minutes to generate the DIR, and another 5 minutes to generate the non-uniform prescribed dose distribution. After the plan was optimized, it took 3 minutes to generate the 4D dose from the apparent dose. 
Table 2 Dosimetric indices of normal structures and PTV50 for 4D dose distributions calculated using the t4Dplan method and the ITV method for the three patients.

\begin{tabular}{cccccccc}
\hline & Parameters & \multicolumn{2}{c}{ Patient 1 } & \multicolumn{2}{c}{ Patient 2 } & \multicolumn{2}{c}{ Patient 3 } \\
& & t4DPlan & ITV & t4DPlan & ITV & t4Dplan & ITV \\
\hline $\begin{array}{c}\text { Total } \\
\text { lung }\end{array}$ & V5 (\%) & 55.4 & 63.9 & 32.2 & 32.7 & 41.0 & 42.5 \\
& V10 (\%) & 30.2 & 35.7 & 24.2 & 25.4 & 25.0 & 26.2 \\
& V20 (\%) & 19.1 & 24.3 & 20.7 & 21.7 & 17.3 & 18.2 \\
& V30 (\%) & 14.8 & 19.8 & 18.3 & 17.6 & 13.0 & 13.4 \\
& Mean (Gy) & 13.2 & 16.3 & 12.8 & 12.9 & 11.3 & 11.8 \\
Spinal & Max (Gy) & 30.1 & 26.3 & 31.6 & 32.3 & 34.0 & 38.0 \\
cord & & & & & & & \\
Heart & Mean (Gy) & 10.8 & 13.4 & 23.8 & 24.2 & 18.8 & 19.0 \\
Stomach & V40 (\%) & & & 8.2 & 21.4 & & \\
& V50 (\%) & & & 2.6 & 10.6 & & \\
& Max (Gy) & & & 54.1 & 62.3 & & \\
& Mean(Gy) & & & 19.3 & 27.3 & & \\
Liver & V30(\%) & & & & & 28.3 & 42.1 \\
& Mean (Gy) & & & & & 22.8 & 31.3 \\
PTV50 & V63 (\%) & 98 & 99.3 & 96.4 & 98.0 & 95.1 & 97.0 \\
& CN & 0.74 & 0.57 & 0.91 & 0.78 & 0.81 & 0.67 \\
& R63 & 0.94 & 0.99 & 0.94 & 0.97 & 0.91 & 0.94 \\
\hline
\end{tabular}

Abbreviations: $R_{63}=$ ratio of volume greater than or equal to $63 \mathrm{~Gy}$ of $4 \mathrm{D}$ dose distribution to AppD; $C N=$ conformity number; $V x=$ the volume of structures received dose $>x \mathrm{~Gy}$; Max = Maximum.

\section{Discussion}

Our findings suggest that the t4Dplan method is an effective means of treatment planning, with features that make it superior to the ITV method, which currently is the most common strategy implemented clinically to compensate for respiration-induced target motion. Essentially, the t4Dplan method uses a smaller PTV while designing a heterogeneous target dose distribution for the planning CT. Because the t4Dplan method accounts for the effects of respiratory motion by adjusting dose within the target, the margin can be reduced relative to that in the ITV method plan, leading to more normal structures sparing. The rationale for the t4Dplan technique is as follows: 4D CT shows that all phases from T30 to T70 are usually similar to the T50 phase, which indicates that patients spent more time in the end-exhalation phase than in any other, that is, the tumor stays around the T50 position for a long period, while it remains at other positions, such as T0, for only short periods. The ITV method envelopes the tumor location in 10 phases to generate the treatment target, which means it weights the time that the tumor is in its T0 position the same as the time it is near its T50 position. The consequence is that the planned dose to the tumor's location around the T0 phase may be delivered to normal structures most of the time since the target moved out of the planned position most of time. The strategy of the t4Dplan method is to deliver a smaller dose to the tumor when the tumor is at its T0 position and then deliver higher doses to the tumor when it is close to its T50 position, which will compensate for the underdosing at the T0 position. In this way, normal structure sparing is maximized as the free-breathing patient undergoes radiotherapy.

Methods of designing treatment plans with non-uniform dose distributions to achieve better normal tissue sparing have been tested by several groups. For example, Li et al [23] used a simplified 4D dose calculation method to design a treatment plan with non-uniform dose. This method will only provide a treatment plan with non-uniform dose distribution. The simplified 4D dose calculation method used by Li et al (2006) directly convolves the $3 \mathrm{D}$ dose distribution with a probability distribution of the tumor over the breathing cycle and therefore does not accurately reflect the effects of breathing motion on the dose distribution. In t4Dplan method, the non-uniform target dose in the planning CT dataset is the apparent dose in the target, while the $4 \mathrm{D}$ dose is essentially uniform. The final result presented to the radiation oncologist yields a uniform dose distribution, and the plan is easily adopted by most practitioners. As the current report shows, the t4Dplan method can be readily implemented in the treatment planning system. We expect that this method will be readily adopted in centers where $4 \mathrm{D} C \mathrm{CT}$ scanners and related treatment planning systems are already available.

Other 4D planning approaches which designed the plans on mid-ventilation, mid-position scan or maximum- and minimum-intensity projection to account for the organ motion have been extensively studied by Wolthaus [24], Cuijipers [25] and Guckenberger [26]. According to their studies, a good dose coverage was still obtained even if the tumor was only fully within the prescribed iso-dose line during a small part of the breathing cycle. Therefore, a better normal tissue sparing was achieved compared to the ITV approach that overestimated the margins necessary for the breath motion. The t4Dplan which designs the non-uniform dose agrees with those previous studies. Cuijipers et al (2010) proposed to use a dosimetric margin, $80 \%$ isodose line of the prescribed dose which fully covers the PTV, to reduce margin compared to ITV approach. The coverage of $80 \%$ dose to the PTV for the three patients using t4Dplan was 91\%, 96\% and 90\% respectively. Considering the fact that our t4Dplan is a motion adapted plan, in which the instantaneous hot and cold spots in the dose distribution delivered during various phases of the target motion are specially designed to compensate each other, the dosimetric margin derived using our approach is even smaller than that proposed by 


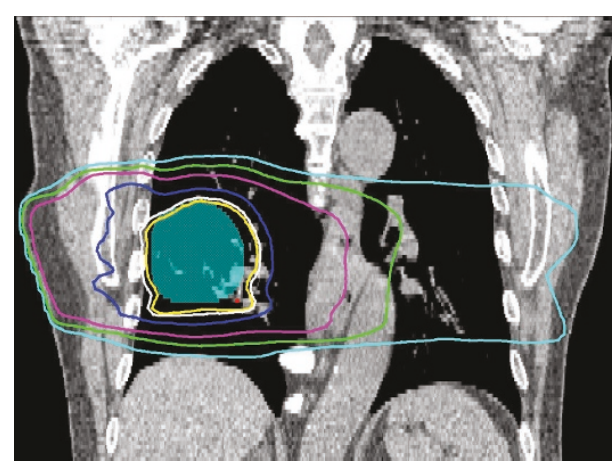

t4Dplan

(a)

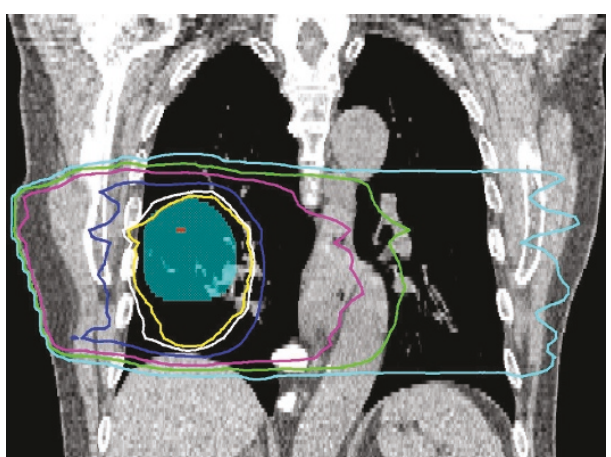

ITV

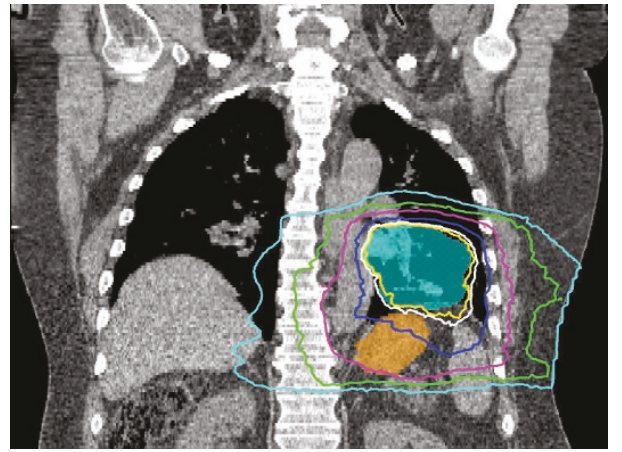

t4Dplan

(c)

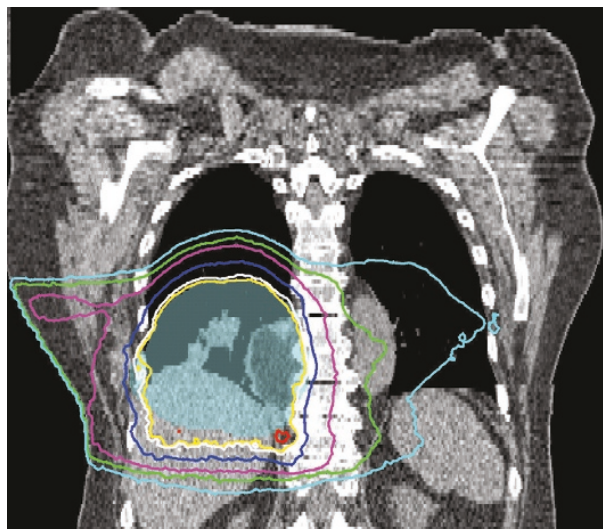

t4Dplan

(e)
Patient 1

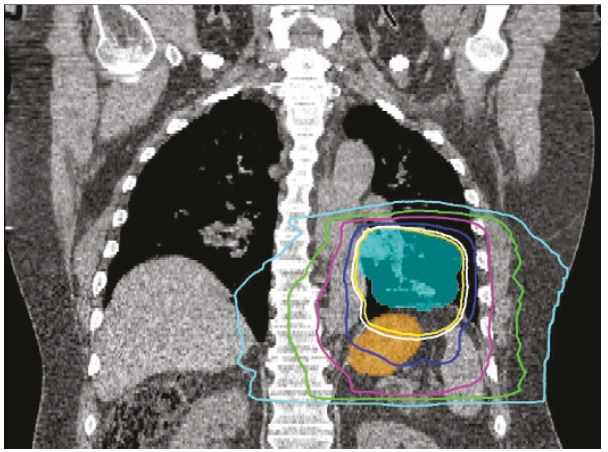

ITV

(d)

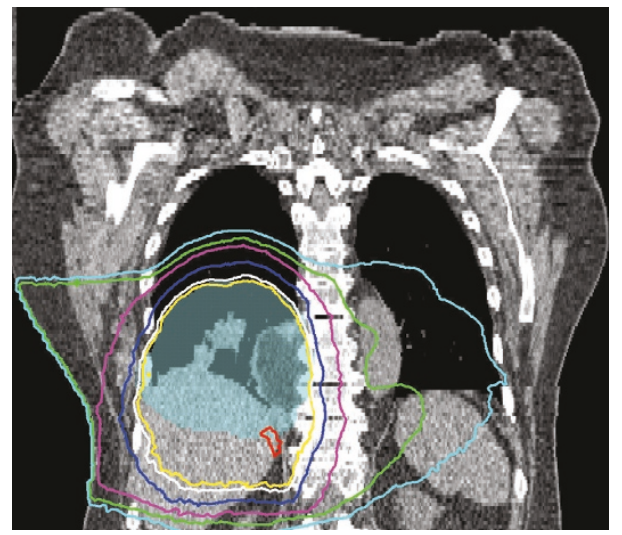

ITV

Patient 2
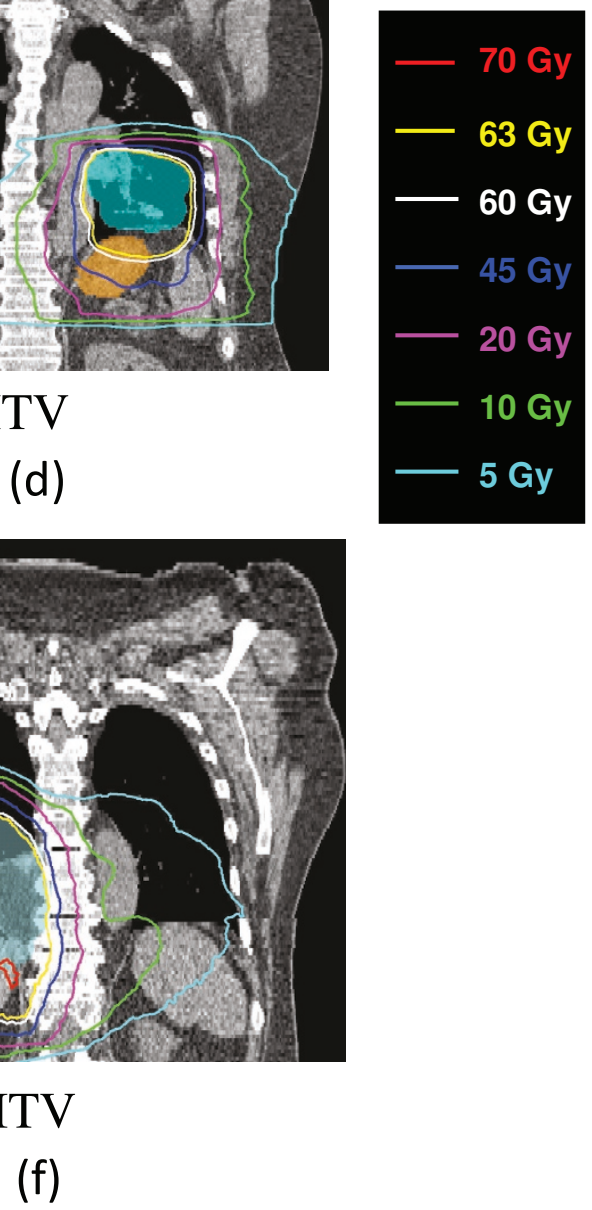

(f)

Figure 6 4D dose distributions calculated using the t4Dplan method for (a) patient 1, (c) patient 2 and (e) patient 3, and the ITV method for (b) patient 1, (d) patient 2 and (f) patient $\mathbf{3}$. The blue color-wash represents the PTV50. The orange color-wash represents 


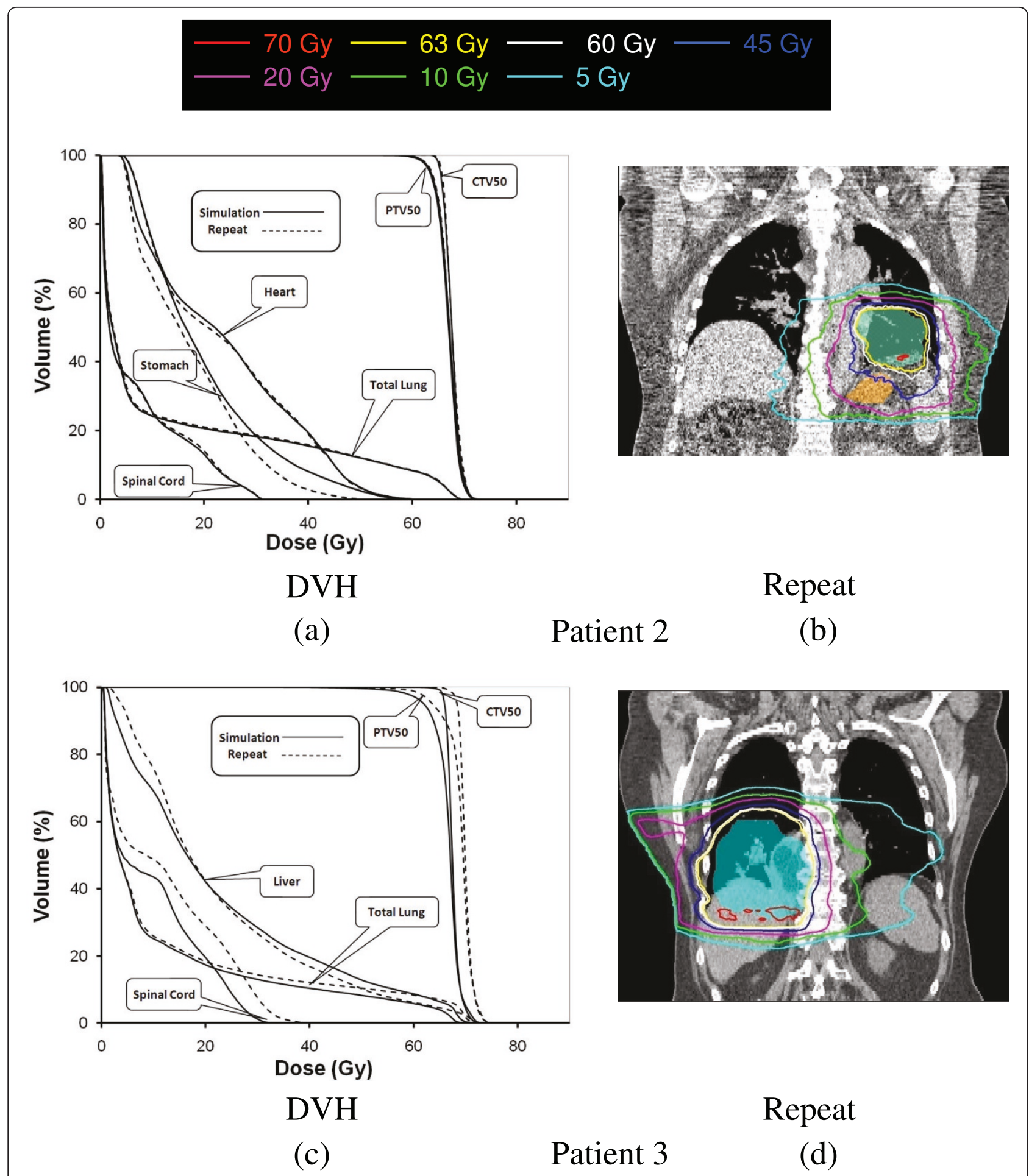

Figure 7 (a) DVHs from 4D dose distribution calculated using the t4Dplan method on the simulation CT (solid line) and repeat CT (dashed line) for (a) patient 2 and (c) patient 3;4D dose distribution images calculated using the t4Dplan method on the repeat CT for (b) patient 2 and (d) patient 3. The blue color-wash represents the PTV50. The orange color-wash represents stomach. 
Cuijipers. In this sense, the t4Dplan is a further improvement. One major difference between ours and other works is that, although PTV is underdosed in the apparent dose distribution, PTV50 is adequately covered in 4D dose. Evaluating the coverage of PTV50 and normal tissue sparing on 4D dose distribution is much more easily accepted by physicians and a natural transition from ITV approach to the t4Dplan approach.

Our t4Dplan method essentially converts the 4D planning problem to a dose-painting problem based on 4D anatomic information. Currently, the dose-painting problem is a subject of intense research in the radiotherapy community [27-32]. The robustness of plans designed by dose-painting algorithms is widely recognized as a major challenge to advancing the technique [33]. In our study, we recalculated the plan designed using the simulation 4D CT by using a repeat 4D CT that exhibited the irregularity of breathing motion to test the robustness of our method. We found that our method is relatively robust against the irregularity of breathing motion for the two patients. One explanation is that our method did not require "exact" dose painting of the voxelized dose prescription. As shown in figure 5(a), (c) and $5(\mathrm{e})$, as long as the hot regions were "painted," the underdose due to the smaller margin would be compensated for. The other explanation was pointed by Cuijipers et al. (2010). There are two uncertainties affecting the robustness of any $4 \mathrm{D}$ planning method: 1 ) the interfractional variability of tumor motion due to the changes of the tumor motion in the breathing pattern of the patient and 2) and changes in the mean tumor position and tumor volume during the course of the treatment. As realized by Cuijipers et al. (2010) for breathing amplitude of $15 \mathrm{~mm}$, a 30\% change in amplitude corresponds to a change in a breathing margin of about 1 $\mathrm{mm}$, implying that the impact of the first uncertainties is expected to be small. The second uncertainty is also small for most patients [25] and is also not considered in the PTV/ITV approach.

Other techniques, such as respiratory gating and breath hold, also showed critical organ sparing compared to ITV approach [34,35]. But for those patients who could not comply with the breath hold technique, our t4Dplan method and respiratory gating may be the good candidates to treat. Compared to the gating method, the t4Dplan does not require 4D delivery, instead, delivers the dose continuously and saves a lot of beam time, with the price of increasing around $15 \mathrm{~min}$ utes in the planning time. Considering the current treatment planning which required many rounds of trial and error, the increase in planning time is almost negligible. Although we demonstrated that our method was relatively robust using two patients as an example, in the clinical practice adopting our method, a motion monitoring protocol and adaptive planning strategy are suggested to ensure the target was adequately covered. For example, the 4D repeat CT should be taken and the robustness of the plan should be checked using the same approach for patients 2 and 3 . If the target was found not adequately to be covered due to irregular breathing pattern, the new adaptive plan should be designed based on the new repeat 4D CT.

Of note, the three patient datasets used in this study illustrate the t4Dplan method as well as demonstrate the effectiveness and robustness of $\mathrm{t} 4 \mathrm{Dplan}$ method. A study of applying this method to a cohort of patients is undergoing and summary of the study will be presented in near future.

\section{Conclusions}

The t4Dplan method is an effective and practical method for designing 4D treatment plans for tumors subject to respiratory motion. The t4Dplan method creates plans that permit better sparing of the normal structures than the commonly used ITV method, which overcompensates for the dosimetric effects of respiration-induced motion to the target. The t4Dplan method does not require 4D treatment delivery and therefore can be readily adopted in centers where 4D CT scanning is already available.

\section{Acknowledgements}

The authors thank Karl Bzdusek and Michael Kaus from Philips Medical Systems for technical support on the research version of the Pinnacle ${ }^{3}$ treatment planning system (8.1x). We thank Professor John Wong from John Hopkins University for spurring us the idea of using non-uniform dose distribution to design 4D lung treatment plan during his visit at MD Anderson. We also want to thank Professor Robert Jeraj from the University of Wisconsin for the helpful discussion on the robustness of dose-painting problems during his visit at MD Anderson this year. This work was presented at the fifty-first conference of the American Association of Physicists in Medicine as a moderated poster discussion. This work is partially supported by NIH grant 16672 .

\section{Authors' contributions}

All authors read and approved the final manuscript. XZ originated the idea, $\mathrm{XL}$ and $\mathrm{XW}$ carried out all the $\mathrm{CT}$ evaluation, target delineation. $\mathrm{XL}$ also drafted the manuscript. $X L$ and $Y L$ contributed to the acquisition of the data and the plan optimization. $X W$ and $X Z$ contributed to the final version of the manuscript.

\section{Competing interests}

The authors declare that they have no competing interests.

Received: 4 May 2011 Accepted: 19 July 2011 Published: 19 July 2011

\section{References}

1. Rietzel E, Chen GTY, Choi NC, Willet CG: Four-dimensional image-based treatment planning: Target volume segmentation and dose calculation in the presence of respiratory motion. International Journal of Radiation Oncology Biology Physics 2005, 61:1535-1550.

2. Keall P: 4-dimensional computed tomography imaging and treatment planning. Seminars in Radiation Oncology 2004, 14:81-90.

3. Keall PJ, Mageras GS, Balter JM, Emery RS, Forster KM, Jiang SB, Kapatoes JM, Low DA, Murphy MJ, Murray BR, et al: The management of 
respiratory motion in radiation oncology report of AAPM Task Group 76. Medical Physics 2006, 33:3874-3900.

4. Low DA, Nystrom M, Kalinin E, Parikh P, Dempsey JF, Bradley JD, Mutic S, Wahab SH, Islam T, Christensen G, et al: A method for the reconstruction of four-dimensional synchronized CT scans acquired during free breathing. Medical Physics 2003, 30:1254-1263.

5. Mageras GS, Pevsner A, Yorke ED, Rosenzweig KE, Ford EC, Hertanto A, Larson SM, Lovelock DM, Erdi YE, Nehmeh SA, et al: Measurement of lung tumor motion using respiration-correlated CT. International Journal of Radiation Oncology Biology Physics 2004, 60:933-941.

6. Trofimov A, Rietzel E, Lu HM, Martin B, Jiang S, Chen GTY, Bortfeld T: Temporo-spatial IMRT optimization: concepts, implementation and initial results. Physics in Medicine and Biology 2005, 50:2779-2798.

7. Werner R, Ehrhardt J, Frenzel T, Saring D, Lu W, Low D, Handels H: Motion artifact reducing reconstruction of $4 \mathrm{D} C \mathrm{CT}$ image data for the analysis of respiratory dynamics. Methods of Information in Medicine 2007, 46:254-260.

8. Wolthaus JWH, Schneider C, Sonke JJ, van Herk M, Belderbos JSA, Rossi MMG, Lebesque JV, Damen EMF: Mid-ventilation CT scan construction from four-dimensional respiration-correlated CT scans for radiotherapy planning of lung cancer patients. International Journal of Radiation Oncology Biology Physics 2006, 65:1560-1571.

9. Yamamoto T, Langner U, Loo BW, Shen J, Keall PJ: Retrospective Analysis of Artifacts in Four-Dimensional CT Images of 50 Abdominal and Thoracic Radiotherapy Patients. International Journal of Radiation Oncology Biology Physics 2008, 72:1250-1258.

10. Kang YX, Zhang XD, Chang JY, Wang H, Wei X, Liao ZX, Komaki R, Cox JD, Balter PA, Liu H, et al: 4D proton treatment planning strategy for mobile lung tumors. International Journal of Radiation Oncology Biology Physics 2007, 67:906-914.

11. Shimizu S, Shirato H, Ogura S, Akita-Dosaka H, Kitamura K, Nishioka T, Kagei K, Nishimura M, Miyasaka K: Detection of lung tumor movement in real-time tumor-tracking radiotherapy. International Journal of Radiation Oncology Biology Physics 2001, 51:304-310.

12. Remouchamps VM, Vicini FA, Sharpe MB, Kestin LL, Martinez AA, Wong JW: Significant reductions in heart and lung doses using deep inspiration breath hold with active breathing control and intensity-modulated radiation therapy for patients treated with locoregional breast irradiation. International Journal of Radiation Oncology Biology Physics 2003, 55:392-406.

13. Yorke ED, Wang L, Rosenzweig KE, Mah D, Paoli JB, Chui CS: Evaluation of deep inspiration breath-hold lung treatment plans with Monte Carlo dose calculation. International Journal of Radiation Oncology Biology Physics 2002, 53:1058-1070.

14. Suh YL, Weiss E, Zhong HL, Fatyga M, Siebers JV, Keall PJ: A deliverable four-dimensional intensity-modulated radiation therapy-planning method for dynamic multileaf collimator tumor tracking delivery. International Journal of Radiation Oncology Biology Physics 2008, 71:1526-1536.

15. Suh $Y L$, Sawant $A$, Venkat $R$, Keall PJ: Four-dimensional IMRT treatment planning using a DMLC motion-tracking algorithm. Physics in Medicine and Biology 2009, 54:3821-3835.

16. Gui MZ, Feng YM, Yi BY, Dhople AA, Yu C: Four-dimensional intensitymodulated radiation therapy planning for dynamic tracking using a direct aperture deformation (DAD) method. Medical Physics 2010, 37:1966-1975.

17. Rosu M, Balter JM, Chetty IJ, Kessler ML, McShan DL, Balter P, Ten Haken RK How extensive of a $4 \mathrm{D}$ dataset is needed to estimate cumulative dose distribution plan evaluation metrics in conformal lung therapy? Medical Physics 2007, 34:233-245.

18. Lujan AE, Larsen EW, Balter JM, Ten Haken RK: A method for incorporating organ motion due to breathing into $3 \mathrm{D}$ dose calculations. Medical Physics 1999, 26:715-720.

19. Zhu L, Lee L, Ma YZ, Ye YY, Mazzeo R, Xing L: Using total-variation regularization for intensity modulated radiation therapy inverse planning with field-specific numbers of segments. Physics in Medicine and Biology 2008, 53:6653-6672

20. Pan T, Lee TY, Rietzel E, Chen GTY: 4D-CT imaging of a volume influenced by respiratory motion on multi-slice CT. Medical Physics 2004, 31:333-340.

21. Chang JY, Zhang XD, Wang XC, Kang YX, Riley B, Bilton S, Mohan R, Komaki R, Cox JD: Significant reduction of normal tissue dose by proton radiotherapy compared with three-dimensional conformal or intensity- modulated radiation therapy in Stage I or Stage III non-small-cell lung cancer. International Journal of Radiation Oncology Biology Physics 2006, 65:1087-1096

22. Feuvret L, Noel G, Mazeron JJ, Bey P: Conformity index: A review. International Journal of Radiation Oncology Biology Physics 2006, 64:333-342.

23. Li X, Zhang PP, Mah D, Gewanter R, Kutcher G: Novel lung IMRT planning algorithms with nonuniform dose delivery strategy to account for respiratory motion. Medical Physics 2006, 33:3390-3398.

24. Wolthaus JWH, Sonke JJ, van Herk M, Belderbos JSA, Rossi MMG, Lebesque JV, Damen EMF: Comparison of different strategies to use fourdimensional computed tomography in treatment planning for lung cancer patients. International Journal of Radiation Oncology Biology Physics 2008, 70:1229-1238.

25. Cuijpers JP, Verbakel W, Slotman BJ, Senan S: A novel simple approach for incorporation of respiratory motion in stereotactic treatments of lung tumors. Radiotherapy and Oncology 2010, 97:443-448.

26. Guckenberger M, Kavanagh A, Webb S, Brada M: A novel respiratory motion compensation strategy combining gated beam delivery and mean target position concept - A compromise between small safety margins and long duty cycles. Radiotherapy and Oncology 2011, 98:317-322.

27. Ling CC, Humm J, Larson S, Amols H, Fuks Z, Leibel S, Koutcher JA: Towards multidimensional radiotherapy (MD-CRT): Biological imaging and biological conformality. International Journal of Radiation Oncology Biology Physics 2000, 47:551-560.

28. Alber M, Paulsen F, Eschmann SM, Machulla HJ: On biologically conformal boost dose optimization. Physics in Medicine and Biology 2003, 48:N31-N35.

29. Bentzen SM: Theragnostic imaging for radiation oncology: dose-painting by numbers. Lancet Oncology 2005, 6:112-117.

30. Flynn RT, Bowen SR, Bentzen SM, Mackie TR, Jeraj R: Intensity-modulated x-ray (IMXT) versus proton (IMPT) therapy for theragnostic hypoxiabased dose painting. Physics in Medicine and Biology 2008, 53:4153-4167.

31. Thorwarth D, Eschmann SM, Paulsen F, Alber M: Hypoxia dose painting by numbers: A planning study. International Journal of Radiation Oncology Biology Physics 2007, 68:291-300.

32. Thorwarth D, Soukup M, Atber M: Dose painting with IMPT, helical tomotherapy and IMXT: A dosimetric comparison. Radiotherapy and Oncology 2008, 86:30-34.

33. Jeraj R: personal communication. 2010.

34. Barnes EIA, Murray BR, Robinson DM, Underwood LJ, Hanson J, Roa WHY: Dosimetric evaluation of lung tumor immobilization using breath hold at deep inspiration. International Journal of Radiation Oncology Biology Physics 2001, 50:1091-1098.

35. Engelsman M, Sharp GC, Bortfeld T, Onimaru R, Shirato H: How much margin reduction is possible through gating or breath hold? Physics in Medicine and Biology 2005, 50:477-490.

doi:10.1186/1748-717X-6-83

Cite this article as: Li et al:: A 4D IMRT planning method using deformable image registration to improve normal tissue sparing with contemporary delivery techniques. Radiation Oncology 2011 6:83.

\section{Submit your next manuscript to BioMed Central and take full advantage of:}

- Convenient online submission

- Thorough peer review

- No space constraints or color figure charges

- Immediate publication on acceptance

- Inclusion in PubMed, CAS, Scopus and Google Scholar

- Research which is freely available for redistribution

Submit your manuscript at www.biomedcentral.com/submit
C Biomed Central 